\title{
EFEITO DA VARIAÇÃO DA ESPESSURA DO REVESTIMENTO NO DESEMPENHO DE PAVIMENTOS FLEXÍVEIS AVALIADOS PELO MEPDG
}

\section{Effect of bituminous thickness varying on flexible pavements performance evaluated by the MEPDG}

\author{
Nayara Donelli Pelisson ${ }^{1}$, José Leomar Fernandes Júnior ${ }^{2}$, \\ Carlos Alberto Prado da Silva Júnior ${ }^{3}$, Heliana Barbosa Fontenele ${ }^{4}$ \\ Recebido em 29 de setembro de 2013; recebido para revisão em 01 de outubro de 2013; aceito em 27 de outubro \\ de 2013; disponível on-line em 21 de novembro de 2013.
}

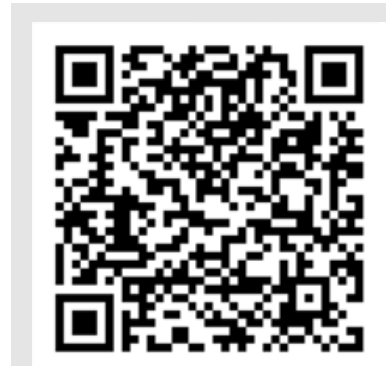

PALAVRAS CHAVE:

MEPDG;

Pavimentos flexíveis;

Dimensionamento de pavimentos.

\section{KEYWORDS:}

MEPDG;

Flexible pavements;

Pavements design.

\begin{abstract}
RESUMO: O dimensionamento de pavimentos de rodovias e de vias urbanas (expressas, arteriais ou coletoras) tem sido realizado em bases empíricas, que apresentam sérias limitações, pois seus resultados são válidos somente para os mesmos, ou muito similares, fatores intervenientes que prevaleciam quando do seu desenvolvimento, há 50 anos. Dessa forma, a AASHTO propôs um novo método para o projeto de pavimentos, cuja base é empírico-mecanística. Tal método, conhecido inicialmente como AASHTO 2002, utiliza para o dimensionamento e previsão do desempenho, os espectros de carga por eixo dos veículos comerciais de carga, além de informações detalhadas sobre o clima e materiais das camadas. No presente trabalho foi realizada uma análise de sensibilidade para investigar o efeito que a variação das espessuras do revestimento acarreta nas respostas estruturais do pavimento flexível, bem como na previsão dlo seu desempenho. Assim, foram feitas simulações com o programa computacional MEPDG utilizando-se espectros de carga desenvolvidos por Fontenele (2011), cujos resultados quantificam a magnitude dos efeitos causados pelos fatores selecionados e suas interações sobre o desempenho final do pavimento e podem, portanto, contribuir para o aperfeiçoamento do dimensionamento de pavimentos e para a diminuição dos insucessos caracterizados pela deterioração precoce de pavimentos rodoviários e urbanos.
\end{abstract}

ABSTRACT: The pavement design of highways and urban roads (freeways, arterials or collectors) has been performed on empirical basis, which present serious limitations, because their results are just valid for the same, or very similar, intervening factors that prevailed at the time of its development, more than fifty years ago. In this way, the AASHTO proposed a new method of pavements design, whose base is mechanisticempirical. This method, which was initially known as AASHTO 2002, uses the axle load spectra of commercial vehicles for design and performance prediction, as well as detailed information about climate and layers materials. This work performed a sensitivity analysis to investigate the effect that thickness variation of bituminous layer can produce to the structural responses of flexible pavements and their effects on pavement performance. Thus, simulations were made with the computer program MEPDG using load spectra developed by Fontenele (2011) and it was quantified the effects magnitude caused by the selected factors and their interaction on the final performance of pavements. Therefore, it can contribute to the improvement of pavement design and the reduction of failures characterized by the early deterioration of highways and urban pavements.

\footnotetext{
* Contato com o autor:

${ }^{1}$ e-mail : nayara_doneli@hotmail.com (N. D. Pelisson)

Aluna de Graduação do Curso de Engenharia Civil da Universidade Estadual de Londrina

2e-mail : leomar@sc.usp.br ( J. L. Fernandes Jr. )

Professor Doutor da Universidade de São Paulo - Escola de Engenharia de São Carlos

${ }^{3}$ e-mail : carlospradojr@hotmail.com ( C. A. P. da Silva Jr. )

Professor Mestre da Universidade Estadual do Oeste do Paraná

${ }^{4}$ e-mail : heliana@uel.br ( H. B. Fontenele)

Professora Doutora da Universidade Estadual de Londrina
} 


\section{INTRODUÇÃO}

Nas últimas décadas estudos têm sido desenvolvidos para buscar representar com maior proximidade à realidade de campo as condições impostas pelo carregamento de veículos e seus efeitos no pavimento. Essas pesquisas visam determinar estruturas eficientes que apresentem comportamento adequado e previsível perante as solicitações de tráfego esperadas durante o período de projeto. Tais estudos têm sua contribuição, inclusive, no Sistema de Gerência de Pavimentos (SGP), com vistas a adequar o emprego de recursos disponíveis a partir da escolha de corretas intervenções e da verificação do momento adequado de ocorrência das mesmas.

O dimensionamento de pavimentos, nos últimos anos, tem sido baseado em métodos de bases empíricas, resultantes de experimentos realizados na década de 60 . Esses métodos têm seu conceito apoiado nas observações da evolução da condição dos pavimentos em campo e os resultados associados a fatores como a resistência dos materiais e a repetição de cargas do tráfego, sendo então a vida remanescente do pavimento expressa pelo número de solicitações equivalentes ao eixo padrão de 8,2 tf. Os métodos de origem empírica apresentam limitações quanto à representação do efeito do tráfego nas estruturas do pavimento, uma vez que os resultados só são reproduzíveis em regiões com as mesmas, ou muito similares, condições naturais daquela na qual ele foi desenvolvido. Tal situação ainda é agravada pela modificação das características dos veículos, como tipo de eixo, tipo de rodagem e pressão de enchimento dos pneus e alteração dos tipos de cargas transportadas; sendo, portanto, muito diferentes das características de cerca de 50 anos atrás.

Em função do exposto, atualmente observa-se a tendência em substituir os métodos puramente empíricos pelos empírico-mecanísticos, em que se relaciona o empirismo de ensaios de campo e laboratoriais com métodos de cálculo mais precisos e realistas, permitindo a determinação das respostas estruturais do pavimento (tensão, deformação e deslocamento). Tais repostas são utilizadas para analisar 0 desempenho dos pavimentos quanto ao surgimento das mais comuns deteriorações (trincas por fadiga e deformações permanentes nas trilhas de roda), prevendo assim os danos acumulados ao longo do período de projeto (Fernandes Jr., 1994; Fontenele, 2011).

A análise do pavimento através do método empírico-mecanístico parte de uma estrutura pré-dimensionada. Com o auxílio de recursos computacionais, as respostas estruturais são determinadas e, através delas, são calculados os danos acumulados durante a vida útil da via. Isso permite estimar e comparar o comportamento do pavimento com os critérios de desempenho estabelecidos para a vida em serviço. Caso estes critérios não sejam obedecidos, é necessário variar os parâmetros de projeto definidos, como as espessuras e as propriedades dos materiais das camadas (módulo de elasticidade e coeficiente de Poisson), até que a condição ideal seja obtida (Fernandes Jr. Paes e Pereira, 2007; Fontenele, 2011).

\subsection{MÉTODO EMPÍRICO-MECANÍSTICO DA AASHTO}

A partir de uma pesquisa do National Cooperative Highway Research Program (NCHRP), patrocinada pela AASHTO (American Association of State Highway and Transportation Officials), foi desenvolvido em 2004 o projeto 1-37 A. No projeto foi apresentado um novo método, de base empírico-mecanística, para o dimensionamento de pavimentos novos e reabilitados. O método ficou conhecido inicialmente como AASHTO 2002, fazendo parte do pacote um software computacional, conhecido como MEPDG (Mechanistic-Empirical Pavement Design Guide). No novo método a forma de caracterização do tráfego foi modificada, sendo então feita a partir da consideração dos espectros de carga por eixo (Axle Load Spectra).

Segundo Haider e Harichandran (2007), os espectros de carga por eixo consistem em informações de carga por eixo e de suas repetições para várias configurações (eixos simples, tandem duplos e triplos), sendo então usados para criar distribuições que representem as cargas dos eixos e as variações a elas associadas. Desta forma, é 
possível esboçar os dados necessários de tráfego de uma maneira mais fiel ao existente em campo.

Assim, o programa MEPDG, inserido no guia de dimensionamento da AASHTO, analisa, a partir de método empírico-mecanístico, o desempenho da estrutura de um pavimento mediante critérios préestabelecidos. Verificando um comportamento indesejado, a conformação da estrutura é reavaliada a partir de variações nos parâmetros de projeto, de forma a buscar sua adequação. 0 procedimento é repetido até que a condição desejada seja alcançada. O software também se utiliza de uma aproximação hierárquica na incorporação das variáveis de entrada, em função da importância do projeto e da disponibilidade dos dados, o que permite ao projetista uma maior flexibilidade. Tal aproximação se refere aos dados de tráfego, materiais e meio ambiente. A Figura 1, adaptada da NCHRP (2004), apresenta um fluxograma que exemplifica sucintamente as etapas de manuseio dos dados e a maneira como estes são interpretados pelo método da AASHTO.

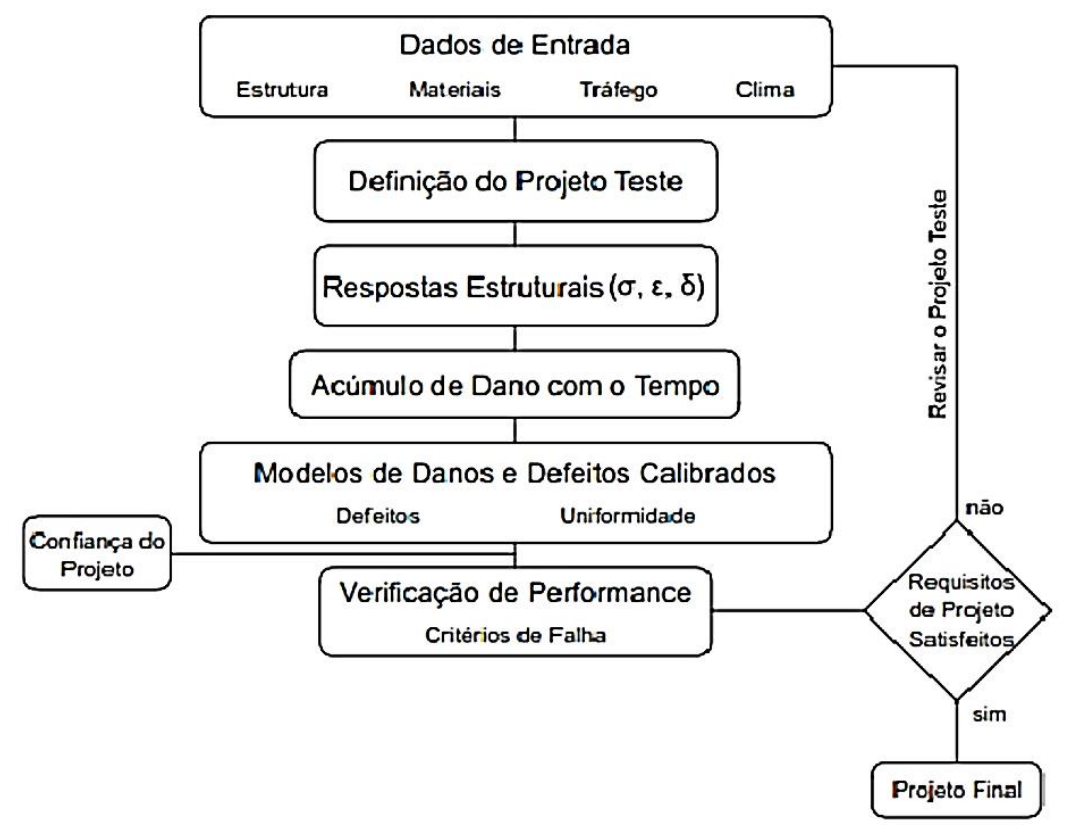

Figura 1: Fluxograma representando o método da AASHTO

Fonte: Adaptado de NCHRP (2004).

\subsection{ANÁLISE DE SENSIBILIDADE}

A partir dos resultados obtidos com o MEPDG, é possível conduzir uma análise de sensibilidade para verificar os efeitos da variação dos parâmetros no comportamento dos pavimentos ao longo de sua vida em serviço. De acordo com Schwartz et al (2013), uma análise de sensibilidade é a distribuição da variabilidade dos dados de saída de um modelo para os seus diversos dados de entrada. Ainda segundo os autores, um conjunto rico e poderoso de técnicas formais e rigorosas para realização da análise de sensibilidade foi desenvolvido ao longo dos últimos 25 anos, podendo ser classificados de diferentes maneiras, como análises locais de sensibilidade ou métodos globais de análise. Seu trabalho foca na análise global de sensibilidade de pavimentos flexíveis para dados de projeto do MEPDG sob cinco condições climáticas e três níveis de tráfego. Os resultados encontrados apontam para o fato de que um dos dados de projeto mais consistentes nas categorias de sensibilidade para todos os defeitos foi a espessura do revestimento asfáltico. As trincas por fadiga e as longitudinais também apresentaram sensibilidade com relação à espessura da base.

Shahji (2006) avaliou a sensibilidade dos parâmetros de dimensionamento de pavimentos flexíveis e rígidos com base no resultados do MEPDG. A análise foi realizada alterando um parâmetro de cada vez, mantendo todos os outros constantes. Em sua pesquisa, o autor variou as 
espessuras em seis valores dentro de uma faixa: do revestimento entre $5 \mathrm{~cm}$ e 17,5 cm (2 a 7 pol); da base entre $12,5 \mathrm{~cm}$ e $25 \mathrm{~cm}$ (5 a 10 pol) e de subbase entre $17,5 \mathrm{~cm}$ e $30 \mathrm{~cm}$ (7 a 12 pol). A partir disso, ele verificou que houve uma maior influência da variação das espessuras do revestimento para a ocorrência dos defeitos.

No trabalho, o autor afirma que o modelo de trincas no revestimento do tipo up-down são menos sensíveis a todos os parâmetros de projeto por ele considerados (tráfego, espessuras e módulos de elasticidade), mas são muito sensíveis à variação da espessura do revestimento asfáltico. Foi observado também que as trincas por fadiga do tipo bottom-up são mais influenciadas pelos parâmetros de tráfego (VDMA) e espessura do revestimento asfáltico e têm pouco efeito pelos outros parâmetros de projeto, como espessura da sub-base e módulo de elasticidade do subleito. A deformação permanente nas trilhas de rodas na camada de revestimento é mais influenciada pelo volume de tráfego e pela espessura do mesmo.

Os resultados da pesquisa de Shahji (2006) levaram à conclusão que as mudanças nas propriedades da base, sub-base e subleito não têm muito impacto na deformação do pavimento, o que o autor salienta como "não muito realista". Com relação à deformação permanente total do pavimento, foi percebido que a maior influência era devido ao tráfego e à espessura da camada asfáltica. $\mathrm{O}$ autor conclui, por fim, que o desempenho do pavimento depende da combinação de espessuras de camada asfáltica e do módulo de subleito usados para o projeto.

\section{OBJETIVO}

Este trabalho tem como objetivo investigar o efeito que a variação da espessura do revestimento acarreta nas respostas estruturais do pavimento flexível, bem como na previsão do seu desempenho.

\section{MÉTODO}

O procedimento desenvolvido nesta pesquisa teve início com a determinação dos parâmetros de projeto a serem variados. Definiu-se para a análise a variação da espessura da camada de revestimento do pavimento, como forma de investigar a influência da mesma com relação às respostas estruturais e, por conseguinte, ao desempenho do pavimento. Posteriormente foram definidos os dados de entrada no programa computacional MEPDG, bem como a determinação dos critérios de desempenho da estrutura analisada.

\subsection{ESTRUTURA DE PAVIMENTO}

As estruturas de pavimento utilizadas nesta pesquisa são apresentadas nas Tabelas 1 a 5.

Tabela 1: Espessuras das camadas, Módulo de Elasticidade e Coeficiente de Poisson

\begin{tabular}{cccc} 
Camada & $\begin{array}{c}\text { Espessura } \\
(\mathbf{c m})\end{array}$ & $\begin{array}{c}\text { Módulo de Elasticidade } \\
\text { (MPa) }\end{array}$ & $\begin{array}{c}\text { Coeficiente } \\
\text { de Poisson }\end{array}$ \\
\hline $\mathbf{1}$ & 7,$5 ; 10,0 ; 12,5 ; 15,0$ & 4.000 & 0,30 \\
$\mathbf{2}$ & 20,0 & 400 & 0,40 \\
$\mathbf{3}$ & 25,0 & 200 & 0,40 \\
Subleito & $\infty$ & 70 & 0,45 \\
\hline
\end{tabular}


Tabela 2: Características da Camada 1 (revestimento)

\begin{tabular}{|c|c|c|c|c|c|c|c|c|}
\hline \multicolumn{9}{|c|}{ Camada 1} \\
\hline $\begin{array}{r}\text { Distr } \\
\text { Granu } \\
\end{array}$ & $\begin{array}{l}\text { ição } \\
\text { iétric }\end{array}$ & & \multirow{2}{*}{\multicolumn{2}{|c|}{ Ligante Asfáltico }} & \multirow{3}{*}{$\begin{array}{c}\begin{array}{c}\text { Vol. de } \\
\text { vazios }\end{array} \\
(\%)\end{array}$} & \multirow{3}{*}{$\begin{array}{c}\begin{array}{c}\text { Densidade da } \\
\text { Mistura }\end{array} \\
\mathrm{Kg} / \mathrm{cm}^{3} \text { (pcf) }\end{array}$} & \multirow{3}{*}{$\begin{array}{c}\text { Condutividade } \\
\text { Térmica do } \\
\text { Asfalto }\end{array}$} & \multirow{3}{*}{$\begin{array}{r}\begin{array}{r}\text { Capacidade } \\
\text { de Calor do } \\
\text { Asfalto }\end{array} \\
\text { BTU/Ib-F }\end{array}$} \\
\hline \multicolumn{3}{|c|}{ Faixa C - DNIT } & & & & & & \\
\hline$\#$ & $\begin{array}{c}\% \\
\text { RA }\end{array}$ & $\begin{array}{l}\% \\
\text { PA }\end{array}$ & Tipo & Teor (\%) & & & & \\
\hline $3 / 4 "$ & 0 & - & & & & & & \\
\hline 3/8" & 20 & - & CAP & & & & & \\
\hline $\mathrm{n}^{\circ} 4$ & 42 & - & $60-70$ & 6 & 4 & $2,5(142)$ & $0,6 /$ & 0,23 \\
\hline$n^{\circ} 200$ & - & 6 & & & & & & \\
\hline
\end{tabular}

Tabela 3: Características da Camada 2 (base)

Camada 2

\begin{tabular}{|c|c|c|c|c|c|}
\hline \multirow{2}{*}{\multicolumn{2}{|c|}{$\begin{array}{c}\begin{array}{c}\text { Distribuição } \\
\text { granulométrica }\end{array} \\
\text { Faixa C - DER/SP }\end{array}$}} & \multirow{3}{*}{\multicolumn{3}{|c|}{ Base }} & \multirow{3}{*}{$\begin{array}{c}\text { Coeficiente de pressão } \\
\text { Lateral }\end{array}$} \\
\hline & & & & & \\
\hline$\#$ & $\%$ PA & & & & \\
\hline $\begin{array}{c}n^{\circ} 4 \\
n^{\circ} 200\end{array}$ & $\begin{array}{l}50 \\
10\end{array}$ & Granular & Brita graduada simples & Compactada & 0,5 \\
\hline
\end{tabular}

Tabela 4: Características da Camada 3 (sub-base)

Camada 3

\begin{tabular}{c|c|c}
\hline \multicolumn{2}{c|}{ Sub-base } & Coeficiente de pressão Lateral \\
\hline Solo A-2-6 & Compactada & 0,5 \\
\hline
\end{tabular}

Tabela 5: Características da Camada 4 (subleito)

Camada 4

\begin{tabular}{c|c|c}
\hline \multicolumn{2}{c|}{ Subleito } & Coeficiente de pressão Lateral \\
\hline Solo A-7-6 & Não compactado (natural) & 0,5 \\
\hline
\end{tabular}


É importante ressaltar que para todas as camadas foi adotado o nível 3 do MEPDG, o qual é considerado como o de menor precisão, sendo utilizados dados médios da região de coleta. Para parâmetros não especificados acima, foram utilizados dados padrões do programa.

\subsection{TRÁFEGO}

Os dados de tráfego foram considerados como nível 1 , ou seja, o de maior precisão e aquele que é usado para o dimensionamento de pavimentos onde as consequências de um colapso precoce podem ser significativas. Foram inseridos no programa valores locais como distribuição de veículos por classe do volume diário médio anual de tráfego (VDMA), fator de crescimento linear, espectros de carga por eixo e número de eixos por caminhão.

Os espectros adotados, relativos à carga por eixo, foram aqueles desenvolvidos no trabalho de Fontenele (2011). Os dados correspondentes aos veículos utilizados para a elaboração dos mesmos pertenciam ao posto de pesagem localizado no $\mathrm{km}$ 28 da Rodovia dos Imigrantes; os quais foram coletados de Janeiro a Dezembro de 2008, com pesagens realizadas durante 24 horas por dia.

O tráfego observado na via foi composto por sete classes de veículos comerciais. As classes, segundo as classificações do DNIT (2007), eram: 2C, $3 C, 2 S 1,2 S 2,2 S 3,3 S 3$ e 3D4. As frequências relativas a cada uma delas na via foram, respectivamente: $8,72 \%$; $7,41 \% ; 8,41 \% ; 11,18 \%$; $27,40 \%, 13,32 \% ; 23,56 \%$.

O VDMA do tráfego utilizado para o ano base de 2009 estava disponível no site do DER-SP, segundo dados fornecidos pela Agência Reguladora de Serviços Públicos Delegados de Transportes do Estado de São Paulo (ARTESP). Esse volume de tráfego foi obtido a partir de contagens na praça de pedágio do $\mathrm{km} \mathrm{32,381} \mathrm{(Piratininga} \mathrm{-} \mathrm{sentido} \mathrm{Sul),} \mathrm{da}$ Rodovia SP 160, e por sensor de tráfego no sentido contrário, no Km 38,04 (Piratininga - sentido norte). Assim, no ano de 2009 o VDMA verificado foi igual a 10.993 veículos/dia. Nos demais anos de projeto (de 2009 a 2028) o volume de tráfego de cada ano foi calculado considerando uma taxa de crescimento do tráfego de $4 \%$ ao ano. A velocidade utilizada foi a padrão do programa, ou seja, de aproximadamente $100 \mathrm{~km} / \mathrm{h}$. A pressão de enchimento dos pneus considerada foi de 120 psi $(827 \mathrm{kPa})$.

\subsection{CLIMA}

Foi considerada a condição climática de Orlando (Flórida- EUA), em função da similaridade dos índices pluviométricos da região onde se encontra a Rodovia dos Imigrantes. Em média, tal índice é de aproximadamente $1.317 \mathrm{~mm}$ de chuva por ano. A profundidade do lençol freático foi de $10 \mathrm{~m}$.

\subsection{CRITÉRIOS DE DESEMPENHO DO PAVIMENTO}

O nível de confiança (segurança) considerado para todos os critérios foi de $90 \%$, uma vez que, conforme é proposto pela NCHRP (2004), o nível de confiança para restaurações e pavimentos novos nas vias interestaduais e nas Freeways em áreas urbanas deve estar entre 85 e $97 \%$ e em áreas rurais entre 80 e $95 \%$.

Os critérios de desempenho considerados são: Irregularidade longitudinal inicial $\left(\left.I R\right|_{\text {ini }}\right)$ com valor igual a aproximadamente $1 \mathrm{~m} / \mathrm{km}(63 \mathrm{in} / \mathrm{mi})$, onde geralmente o valor ideal para pavimentos novos é de 0,79 a 1,18 m/km (50-75 in/mi); Irregularidade longitudinal final $\left(|\mathrm{RI}|_{\text {final }}\right)$ igual a 4 $\mathrm{m} / \mathrm{km}$ (172 in/mi); Trincas longitudinais up-down de $189 \mathrm{~m} / \mathrm{km}$ (1000 ft/mi); Trincas por fadiga classe 3 bottom-up igual a 25\%; trincas transversais térmicas igual a $189 \mathrm{~m} / \mathrm{km} \quad(1000 \mathrm{ft} / \mathrm{mi})$, Afundamento nas trilhas de rodas no CBUQ igual a $6,35 \mathrm{~mm}(0,25 \mathrm{in})$ e Afundamento nas trilhas de roda do pavimento todo igual a $19,5 \mathrm{~mm}(0,75 \mathrm{in})$.

\section{RESULTADOS E DISCUSSÃO}

A partir das curvas de desempenho geradas pelo MEPDG, com a variação da espessura da camada de revestimento asfáltico, foi possível desenvolver uma análise de sensibilidade. Tal análise foi realizada alterando a espessura da camada, mantendo as outras características constantes. 
Na Figura 2 são apresentados os gráficos gerados a partir dos valores previstos para o $20^{\circ}$ ano de projeto. Pode ser observado que todos os valores apresentados ultrapassaram o limite de projeto, exceto os relativos à IRI. A estrutura não foi
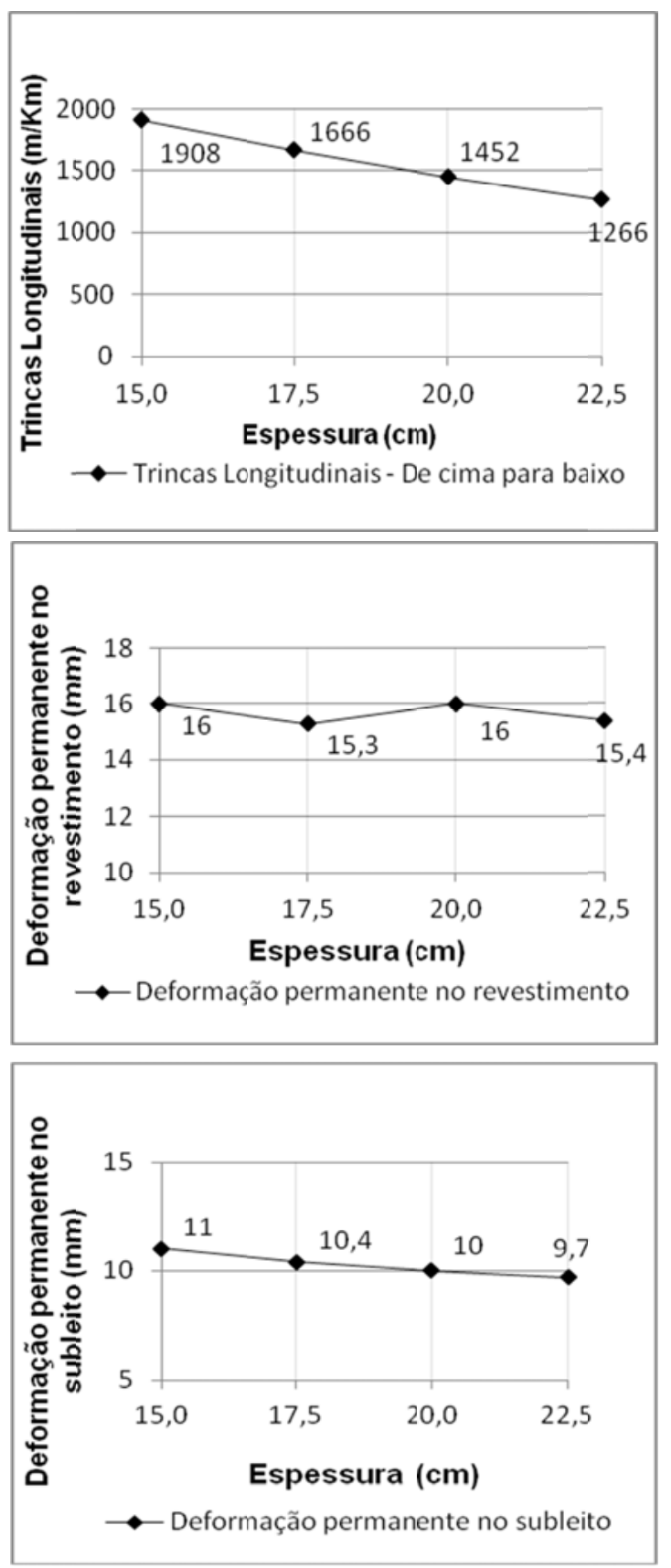

alterada de forma a buscar a adequação em termos de critérios máximos de projeto, tendo em vista que o objetivo desta pesquisa era apenas de verificar a sensibilidade à variação do parâmetro de projeto.
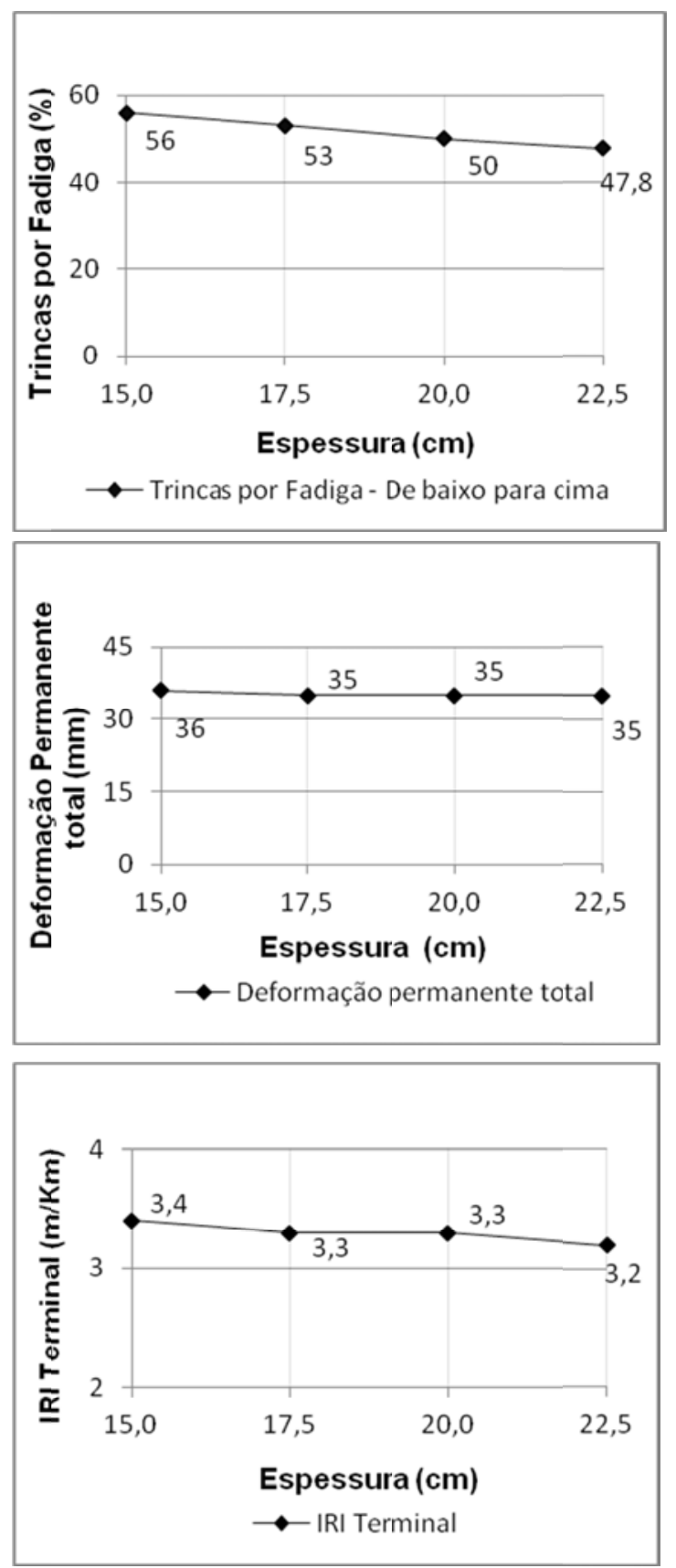

Figura 2: Variação das medidas relativas às deteriorações analisadas para a variação da espessura do revestimento. 
Para as trincas longitudinais (de cima para baixo) é possível verificar que o defeito atinge o ponto de máximo para a espessura de revestimento de $10,0 \mathrm{~cm}$, ou seja, $1.967 \mathrm{~m} / \mathrm{km}$. À medida que se aumenta a espessura da camada, ocorre uma redução do defeito, atingindo um valor mínimo de $663 \mathrm{~m} / \mathrm{km}$ para a espessura de 15,0 cm. A variação de cerca de $66 \%$ do valor mínimo em relação ao máximo revela que o defeito é sensível à variação de espessura da camada. As trincas por fadiga (de baixo para cima), da mesma forma, sofrem uma redução de valores com o aumento da espessura da camada, apresentando um máximo de $81,1 \%$ para $7,5 \mathrm{~cm}$ e um mínimo de $35 \%$ para $15,0 \mathrm{~cm}$. A redução corresponde é igual a 57\%, o que indica que o defeito também é sensível à variação de espessura do revestimento.

As deformações permanentes (apenas no revestimento) atingem seu ponto de máximo (20 $\mathrm{cm}$ ) para a espessura do revestimento de 7,5 cm. Com o aumento da espessura da camada para 15,0 $\mathrm{cm}$ é prevista uma deformação de 14,5 mm (ponto mínimo). A redução de $27,5 \%$ mostra que o defeito em questão é sensível à variação da espessura. Já com relação às deformações permanentes em todo o pavimento, é apresentada uma redução do defeito ao se aumentar a espessura do revestimento. Tem-se o valor máximo de $40 \mathrm{~mm}$ para 7,5 cm de espessura do revestimento e o valor mínimo de $33 \mathrm{~mm}$ para 15,0 cm da camada. Há, então, para as deformações em todo o pavimento uma redução de $17,5 \%$, o que mostra certa sensibilidade do defeito em função da variação da espessura da camada.

Da mesma forma, a deformação permanente apenas no subleito apresenta uma redução com o aumento da espessura do revestimento, havendo uma variação de 14,3\% entre os extremos. $O$ Índice de Irregularidade Internacional (IRI) apresentou valor máximo de $4,2 \mathrm{~m} / \mathrm{km}$ para a espessura de $7,5 \mathrm{~cm}$ do revestimento e mínimo de $3 \mathrm{~m} / \mathrm{km}$ para $15,0 \mathrm{~cm}$ do revestimento. Essa redução de cerca de $29 \%$ indica que a irregularidade longitudinal é sensível à variação da espessura da camada.

\section{CONCLUSÕES}

A partir da análise realizada foi possível verificar que a ocorrência dos defeitos trincas longitudinais, trincas por fadiga e deformações permanentes apenas no revestimento são bem sensíveis quando se varia a espessura do revestimento asfáltico.

Tal situação também ocorreu para a deformação em todo o pavimento, porém apresentando uma menor sensibilidade à variação da espessura do revestimento quando comparada à deformação apenas no revestimento e no subleito. Para o IRI, foi constatada também a sensibilidade com a variação da espessura do revestimento.

Alguns dos resultados do presente trabalho diferem daqueles apresentados por Shahji (2006). Em sua análise de sensibilidade, Shahji (2006) constatou que o aumento da espessura do revestimento só produz uma queda nas formações de trincas de cima para baixo após a espessura de $15,0 \mathrm{~cm}$, bem como nas trincas de baixo para cima após a espessura de $10 \mathrm{~cm}$. Tal discordância pode ser explicada pelo fato do autor ter utilizado algumas características diferentes, como, por exemplo, o tráfego e os materiais.

Um aspecto semelhante observado na presente pesquisa foi com relação às deformações permanentes, sendo essas mais influenciadas pela variação da espessura do revestimento.

Diante do exposto, a espessura do revestimento é um parâmetro que apresenta um efeito considerável, principalmente no que se refere ao defeito trincas. Em relação às trincas longitudinais, percebe-se um ponto de máxima deterioração para a espessura de $10 \mathrm{~cm}$, enquanto que, para as trincas por fadiga do revestimento, os valores máximos ocorrem entre 5 e 7,5 cm.

Confirmam-se, assim, as evidências de estudos anteriores, inclusive os que levaram à adoção, há muito tempo, por parte da AASHTO (1986), de espessura mínima de $12,5 \mathrm{~cm}$ para o revestimento asfáltico de rodovias com tráfego médio, ou seja, correspondente a um número de aplicações do eixo-padrão relativamente baixo 
$\left(>5 \times 10^{6}\right)$. Infelizmente, no Brasil, todas as evidências relacionadas à importância da espessura da camada de revestimento asfáltico têm sido ignoradas, com adoção de valores que, se representam alguma economia no custo de construção, resultam em custos altíssimos de manutenção e reabilitação e, mais ainda, de operação de veículos ao longo da vida em serviço.

\section{AGRADECIMENTOS}

À Fundação Araucária pelo auxílio financeiro à pesquisa (Chamada 14/2011 Protocolo $n^{\circ}$ 24685).

\section{REFERÊNCIAS BIBLIOGRÁFICAS}

AASHTO - AMERICAN ASSOCIATION OF STATE HIGHWAY AND TRANSPORTATION OFFICIALS. Guide for Design of Pavement Structures. Washington, D. C., 1986.

AASHTO - AMERICAN ASSOCIATION OF STATE HIGHWAY AND TRANSPORTATION OFFICIALS. Mechanistic-Empirical Pavement Design Guide: A manual of practice. Interim edition. Washington, D. C., 2008.

DEPARTAMENTO NACIONAL DE INFRAESTRUTURA DE TRANSPORTE - DNIT. Manual de Projeto de Intersecções. 2 ed. Rio de Janeiro, 2005.

FERNANDES JÚNIOR, J. L. Investigação dos Efeitos das Solicitações do Tráfego sobre o Desempenho de Pavimentos. 1994. 293 f. Tese (Doutorado) Pós-graduação em Engenharia Civil - Escola de Engenharia de São Carlos, Universidade de São Paulo, São Carlos, São Paulo, 1994.

FERNANDES JÚNIOR, J. L.; PAES, J. C.; PEREIRA, P. A. A. Avaliação dos efeitos das solicitações do tráfego sobre o desempenho de pavimentos flexíveis portugueses e brasileiros. Revista Pavimentação, Ano II, n.5, PP.28-38. Associação Brasileira de Pavimentação - ABPv, Rio de Janeiro, 2007.

FONTENELE, B. H. Representação do Tráfego de Veículos Rodoviários de Carga através de Espectros de Carga por Eixo e seu Efeito no Desempenho dos Pavimentos. 2011. Tese (Doutorado) - Programa de Pós-Graduação em Engenharia de Transportes - Escola de Engenharia de São Carlos da Universidade de São Paulo.
HAIDER, S. W.; HARICHANDRAN, R. S. Characterizing axle load spectra by using gross vehicle weights and truck traffic volumes. Transportation Research Record: Journal of the Transportation Research Board. TRB 2007 Annual Meeting CD-ROM. Washington, D. C. 2007.

NCHRP - NATIONAL COOPERATIVE HIGHWAY RESEARCH PROGRAM. Guide for MechanisticEmpirical Design of New and Rehabilitated Pavement Structures. Final Report NCHRP 1-37 A. Champaign, Illinois, 2004.

SCHWARTS, W. C.; LI, R.; CEYLAN, H.; KIM, S.; GOPALAKRISHNAN, K. Global Sensitivity Analysis of Mechanistic-Empirical Performance Predictions for Flexible Pavements. Transportation Research Board. TRB 2013 Annual Meeting CD-ROM. Washington, D. C. 2013.

SHAJHI, S. Sensitivity Analysis of AASHTO's 2002 Flexible and Rigid Pavement Design Methods. 2006. Master of Science in the Department of Civil \& Environmental Engineering - College of Engineering and Computer Science - University of Central Florida. Orlando, Florida, 2006. 